\title{
Calidad microbiológica del aire en un Centro Comunitario de Salud Familiar de Talcahuano, Región del Biobío, Chile
}

\author{
Microbiological air quality in a Community Family Health Center of Talcahuano, \\ Biobío Region, Chile
}

\begin{abstract}
Marco Parra-Donoso', Emky Valdebenito-Rolack', Nicole Cid-Maldonado', Mariana Dominguez-Yévenes', Felipe Sanhueza-Gomez², Christopher Salvo², Marta Lopez-Jenssen², Paula Bournas ${ }^{3}$ y Helia Bello-Toledo 1,4
\end{abstract}

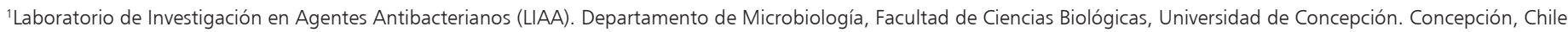
${ }^{2}$ Departamento de Ingeniería de Materiales (DIMAT), Facultad de Ingeniería, Universidad de Concepción. Concepción, Chile.

${ }^{3}$ Centro Comunitario de Salud Familiar Centinela. Talcahuano, Chile.

${ }^{4}$ Millennium Initiative for Collaborative Research on Bacterial Resistance (MICROB-R). Santiago, Chile.
\end{abstract}

Declaración de conflictos de interés: No existen conflictos de interés.

Investigación financiada por Proyecto FONDEF ID 17 i10339.

Recibido: 27 de abril de 2020 (segunda versión: 1 de abril de 2021) / Aceptado: 1 de abril de 2021

\section{Resumen}

Introduccción: La calidad del aire en centros de salud es fundamental para resguardar la salud de las personas. En Chile, los Centros Comunitarios de Salud Familiar (CECOSF) son lugares de gran concurrencia de personas, favoreciendo la diseminación de microorganismos. Objetivo: Evaluar la calidad microbiológica del aire al interior del CECOSF-Centinela en Talcahuano, Región del Biobío. Metodología: Se tomó muestras de aire en seis salas del CECOSF, quincenalmente, entre julio de 2018 y junio de 2019, con el equipo MAS-100 NT, empleando agar tripticasa y agar Sabouraud. Diferentes morfotipos de bacterias y hongos fueron identificados mediante RPC. Resultados: Los recuentos de bacterias y hongos variaron entre $9,1 \times 10^{1}-2,4 \times 10^{3} \mathrm{ufc} / \mathrm{m}^{3}$ y $10-1,5 \times 10^{2} \mathrm{ufc} / \mathrm{m}^{3}$, respectivamente. El aire de la sala de espera presentó los recuentos más altos, tanto para bacterias como hongos $(\mathrm{P}<0,05)$. Se identificó Staphylococcus, Enterococcus, Pseudomonas, Acinetobacter, destacando las especies Staphylococcus aureus y Pseudomonas oryzihabitans, microrganismo este último, descrito actualmente como patógeno nosocomial. Entre los hongos se identificó Aspergillus, Meyerozyma y Rhodotorula. Conclusión: Las muestras de aire del CECOSF-Centinela presentan microrganismos de importancia en salud humana. De ahí la necesidad de formular programas de monitoreo más regulares para controlar la calidad del aire al interior de estos establecimientos.

Palabras clave: calidad microbiológica; aire; contaminación microbiológica; ambiente; contaminación ambiental; carga aérea microbiana.

\section{Abstract}

Background: Indoor air quality in health centers is essential to protect the health of people. In Chile, the Community Family Health Centers (CECOSF) are places with large attendance of people, favoring the dissemination of microorganisms, and there are no reports of the microbial air loading these health centers. Aim: To evaluate the microbiological indoor air quality in CECOSF-Centinela in Talcahuano, Biobío Region. Methods: Air samples were taken in 6 rooms of the CECOSF, every 15 days between July 2018 and June 2019, with the MAS-100 NT equipment using trypticase and Sabouraud agars. Different morphotypes of bacteria and fungi were identified by PCR. Results: The bacterial and fungal counts varied between $9.1 \times 10^{1}-2.4 \times 10^{3} \mathrm{cfu} / \mathrm{m}^{3}$ and $10-1.5 \times 10^{2} \mathrm{cfu} / \mathrm{m}^{3}$, respectively. The air in the waiting room presented the highest counts, both for bacteria and fungi $(\mathrm{P}<0.05)$. Staphylococcus, Enterococcus, Pseudomonas, Acinetobacter were identified, highlighting the species Staphylococcus aureus and Pseudomonas oryzihabitans, the latter described as a nosocomial pathogen. Among the fungi, Aspergillus, Meyerozyma and Rhodotorula were identified. Conclusion: The indoor air of the CECOSF-Centinela presents microorganisms of importance in human health. Therefore, it is necessary to formulate more regular monitoring programs for the control of air quality inside these health centers.

Keywords: microbiological quality; air; microbial contamination; environment; environmental pollution; microbial air load. 


\section{Introducción}

$$
\mathrm{L}
$$

a calidad del aire al interior de los centros de salud, de acuerdo a la Organización Mundial de la Salud (OMS), constituye un factor de riesgo importante para la salud humana, tanto en países de bajos ingresos como de ingresos medios y altos, incidiendo directamente en la calidad de vida de las personas ${ }^{1}$. El aire contiene en suspensión diferentes tipos de microorganismos, especialmente bacterias y hongos; aunque principalmente éstos no son patógenos; también es posible encontrar microrganismos patógenos, que pueden afectar a personas que son particularmente vulnerables debido a su estado de salud o edad ${ }^{2}$. Actualmente, la Organización Internacional para la Estandarización (ISO) no ha establecido el rango aceptable de carga microbiana en el aire de ambientes clínicos. Sin embargo, considerando la importancia de la calidad microbiológica del aire, algunos países han establecidos recomendaciones de carga microbiana aérea máxima, especialmente, en áreas hospitalarias muy críticas como son los pabellones quirúrgicos ${ }^{3-6}$. No obstante, existe escasa información de la calidad del aire en otros ambientes clínicos, no necesariamente hospitalarios.

En Chile, existe una red de establecimientos asistenciales públicos, cuyas funciones y normas son dictadas por el Ministerio de Salud, que están organizados de acuerdo a su cobertura poblacional y complejidad asistencial ${ }^{7}$. Entre estos establecimientos se tiene a los Centros Comunitario de Salud Familiar (CECOSF), que forman parte de la red de Atención Primaria de Salud, cuya misión es brindar atención bajo el Modelo de Salud Familiar y Comunitario $^{7}$. Estos establecimientos son lugares de gran concurrencia de personas, favoreciendo la diseminación de microorganismos, inclusive patógenos, que pueden llegar a contaminar el aire, superficies, dispositivos médicos que contactan la piel o mucosas de las personas que concurren a estos centros, representando un riesgo no sólo para ellos, sino que también para el personal de atención de salud . Además, en Chile, no existe una normativa que indique los límites de carga microbiológica máxima para el aire de estos centros de salud, como tampoco literatura técnica que entregue información de la situación microbiológica en éstos. Por ello, objetivo de este trabajo fue cuantificar e identificar las bacterias y hongos presentes en el aire del CECOSF-Centinela en Talcahuano, Región del Biobío.

\section{Materiales y Métodos}

\section{Sitio de muestreo}

Este estudio fue realizado en el CECOSF ubicado en el cerro Centinela en Talcahuano, Región del Biobío, el que está destinado atender una población de aproximadamente 5.000 personas. El edificio de un piso tiene una superficie de $258 \mathrm{~m}^{2}$ y se distribuye en seis áreas: (A): sala de espera, (B): sala de reuniones, (C): sala de atención odontológica, (D): sala de atención médica general, (E): sala de atención de obstetricia/ginecología y (F): sala de procedimientos generales. La distribución de las salas se presenta en la Figura 1.

\section{Procedimiento de muestreo de aire}

Durante julio de 2018 hasta junio de 2019 se tomaron muestra de aire en seis áreas del CECOSF-Centinela en Talcahuano, Región del Biobío. El muestreo se realizó cada 15 días de manera activa empleando el equipo MAS-100 NT (Merck, Alemania, $\mathrm{n}^{\circ}$ de serie: 15986), equipo recomendado en la norma chilena para el estudio de calidad microbiológica de aire ${ }^{8}$. El equipo se dispuso en el centro de cada sala a una altura de $1 \mathrm{~m}$ y se programó para filtrar $250 \mathrm{~L}$ de aire a un flujo de $100 \mathrm{~L} / \mathrm{min}$. El aire filtrado impactó en placas de agar tripticasa (AT)

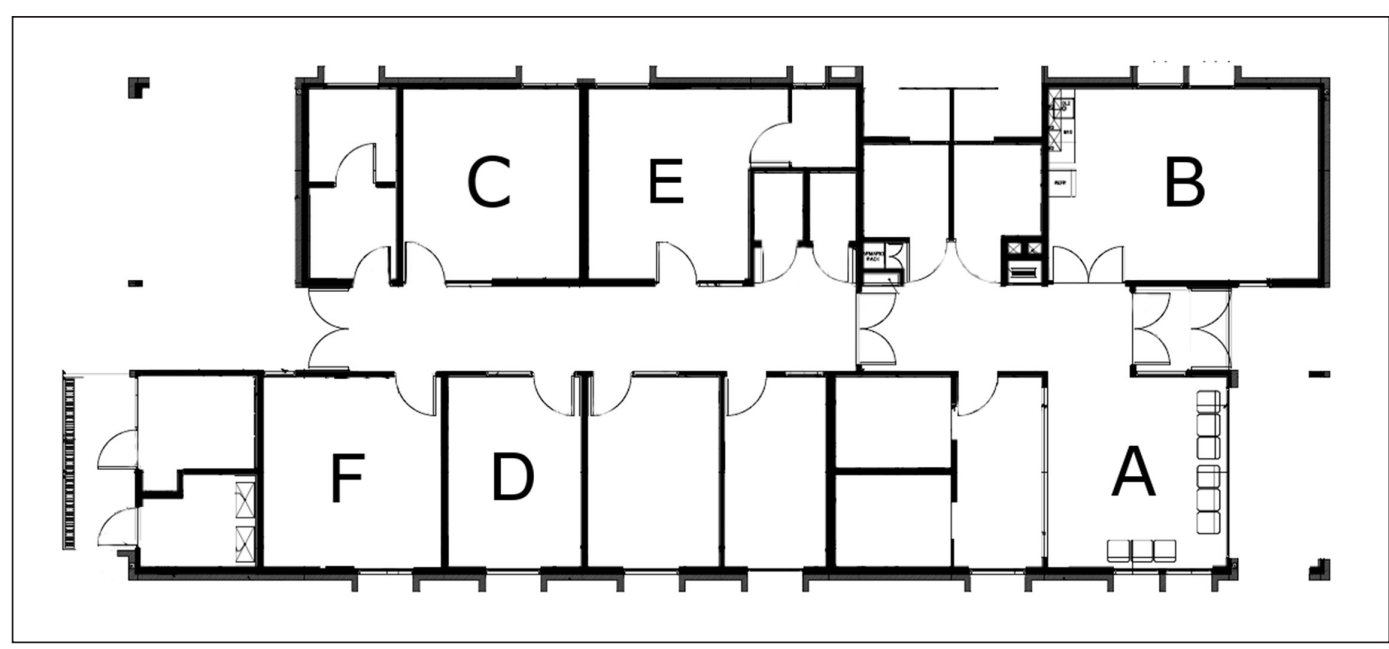

Figura 1. Plano del CECOSF-Centinela de Talcahuano en el que se muestra la disposición de las salas: A: sala de espera, B: sala de reuniones, C: sala de atención odontológica, D: sala de atención médica general, E: sala de atención de obstetricia/ginecología y F: sala de procedimientos generales La entrada al recinto se encuentra entre la sala de espera y reuniones. 
(adicionadas de cicloheximida $2,5 \mu \mathrm{g} / \mathrm{mL}$ ) y placas de agar Sabouraud (AS) (adicionadas de cloranfenicol 0,25 $\mu \mathrm{g} / \mathrm{mL}$ ). Las placas de AT fueron incubadas a $37^{\circ} \mathrm{C}$ por $48 \mathrm{~h}$ y las de $\mathrm{AS}$ a $25^{\circ} \mathrm{C}$ durante 4 días. Los muestreos fueron realizados por triplicado. La medición de temperatura $(\mathrm{T})$ y humedad relativa $(\mathrm{HR})$ se realizó mediante termohigrómetro HI 9564 (Hanna Instruments, USA).

\section{Recuento de microorganismos viables}

Considerando que el equipo muestreador de aire MAS-100 NT (Merck, Alemania, $\mathrm{n}^{\circ}$ de serie: 15986), utiliza una tapa con septo de 300 orificios, el número de colonias de bacterias y hongos, aisladas en las placas de agar, fue corregido empleando la expresión de Feller ${ }^{8}$. Los recuentos fueron expresados por cada metro cúbico de aire muestreado $\left(\mathrm{ufc} / \mathrm{m}^{3}\right)$.

\section{Análisis estadístico}

La distribución de los datos de recuento bacteriano ( $\mathrm{ufc} / \mathrm{m}^{3}$ ) fue determinada mediante la prueba de normalidad D'Agostino y Pearson, con intervalo de confianza de $95 \%$ y $\alpha=0,05$ empleando el software Prism 7 (Graph Pad, USA, 2016). Se consideró que los datos tuvieron una distribución normal cuando el valor de $\mathrm{P}$ fue mayor a 0,05 . El recuento mínimo y máximo por cada sala fue calculado mediante función de autosuma en Excel 15.13 (Micrososft, USA, 2015). Para realizar comparaciones múltiples de carga microbiana entre las salas (promedio) y en relación a la fecha de muestreo, se utilizó prueba ANOVA de dos vías (a través de test de Tukey) cuando la distribución de los datos fue normal, y se usó test de Kruskall-Wallis cuando la distribución de los datos no fue normal. Para determinar la relación entre la carga microbiológica y factores ambientales como T, HR y el número de personas que transitaron en el recinto, se utilizó una muestra de 19 mediciones, por triplicado, empleando el test de correlación no paramétrico de Spearman (parámetro rho; $\rho$ ), empleando el software Prism 7 (Graph Pad, USA). Se consideró que existió correlación en el mismo sentido cuando: $0<\rho<1$; y en el sentido inverso cuando $-1<\rho<0$. Se asumió que no hubo correlación si $\rho=0$. De igual manera, si $\rho$ tuvo un valor distinto de 0 , se consideró que esta correlación no fue al azar sólo si el valor estadístico $\mathrm{P}$ para el test fue $<0,05$ (intervalo de confianza de $95 \%, \alpha=0,05$, dos colas).

\section{Selección e identificación de microorganismos}

A partir de las placas de recuentos, se seleccionaron 100 colonias de bacterias y hongos, con diferentes morfotipos, las que fueron almacenadas en cepario a $-80^{\circ} \mathrm{C}$. Su identificación se realizó mediante reacción de polimerasa en cadena (RPC), utilizando partidores específicos para el ADNr $16 \mathrm{~S}$ y para regiones espaciadoras del transcrito interno del $\mathrm{ADNr}(18 \mathrm{~S}, 5,8 \mathrm{~S}$ y 28S), respectivamente, y siguiendo protocolos previamente descritos ${ }^{9,10}$. Los productos de amplificación fueron enviados a la compañía MacroGen Corp (Seneca Highway, Rockville, Korea) para su secuenciación en ambos sentidos. Las secuencias de ADN editadas fueron sometidas a herramienta de búsqueda por alineamiento básico local BLAST ${ }^{11}$.

\section{Resultados}

\section{Recuento de bacterias y hongos}

Los valores de recuento de bacterias y hongos obtenidos durante julio 2018 a junio 2019 en cada sala del CECOSF-Centinela se presentan en las Figuras 2 y 3, respectivamente. Puede observarse que, durante todo el período muestreado, en las salas A (sala de espera) y D (sala de atención médica general), se encontraron los recuentos bacterianos significativamente mayor en relación a las otras salas $(\mathrm{P}<0,05)$ (Figura 2, asteriscos rojos). Inclusive, en la sala A se obtuvo el recuento bacteriano más alto $\left(2.376 \mathrm{ufc} / \mathrm{m}^{3}\right)$, durante el mes de julio 2018 , que corresponde a temporada de invierno (Tabla 1). Por otra parte, los recuentos de hongos (Figura 3) también alcanzan valores significativamente más altos en temporada otoño-invierno (julio de 2018 y marzo-mayo de 2019) que en primavera-verano (agosto de 2018-enero de 2019) $(\mathrm{P}<0,05)$, encontrándose el valor más alto en la sala $\mathrm{A}$ (recuento máximo de $153 \mathrm{ufc} / \mathrm{m}^{3}$, Tabla 1 ).

\section{Variables ambientales en sala}

En la Tabla 2 se presentan los valores máximos y mínimos de T, HR y número de personas $(\mathrm{N})$ medidos en cada sala. La T mayor $\left(29,3^{\circ} \mathrm{C}\right)$ se obtuvo en la sala B (octubre de 2018 ) y la $\mathrm{T}$ mínima $\left(16^{\circ} \mathrm{C}\right)$, se dio en la sala A (mayo de 2019). En cambio, la HR máxima (81,8\%) se encontró en la sala D (junio de 2019) y la HR mínima (30,5\%), en la sala B (octubre de 2018). Por otra parte, el mayor $\mathrm{N}$, al momento del muestreo, se encontró en la sala A (24 personas), mientras que el mínimo de personas se detectó en las salas C, D, E y F.

\section{Correlación entre variables ambientales y recuento de bacterias y hongos}

En la mayoría de los casos, las variables ambientales como T y HR no tuvieron una correlación significativa con el recuento bacteriano, ni fúngico $(\mathrm{P}>0,05)$. Sólo se observó correlación significativa entre el $\mathrm{N}$ y el recuento de bacterias en la sala $\mathrm{D}(\rho 0,71 ; \mathrm{P}=0,0006)$ y en la $\mathrm{E}$ $(\rho=0,49 ; P=0,0299)$.

\section{Identificación de microorganismos}

La identificación de las cepas de bacterias y hongos se presenta en las Tablas 3 y 4, respectivamente. En el 


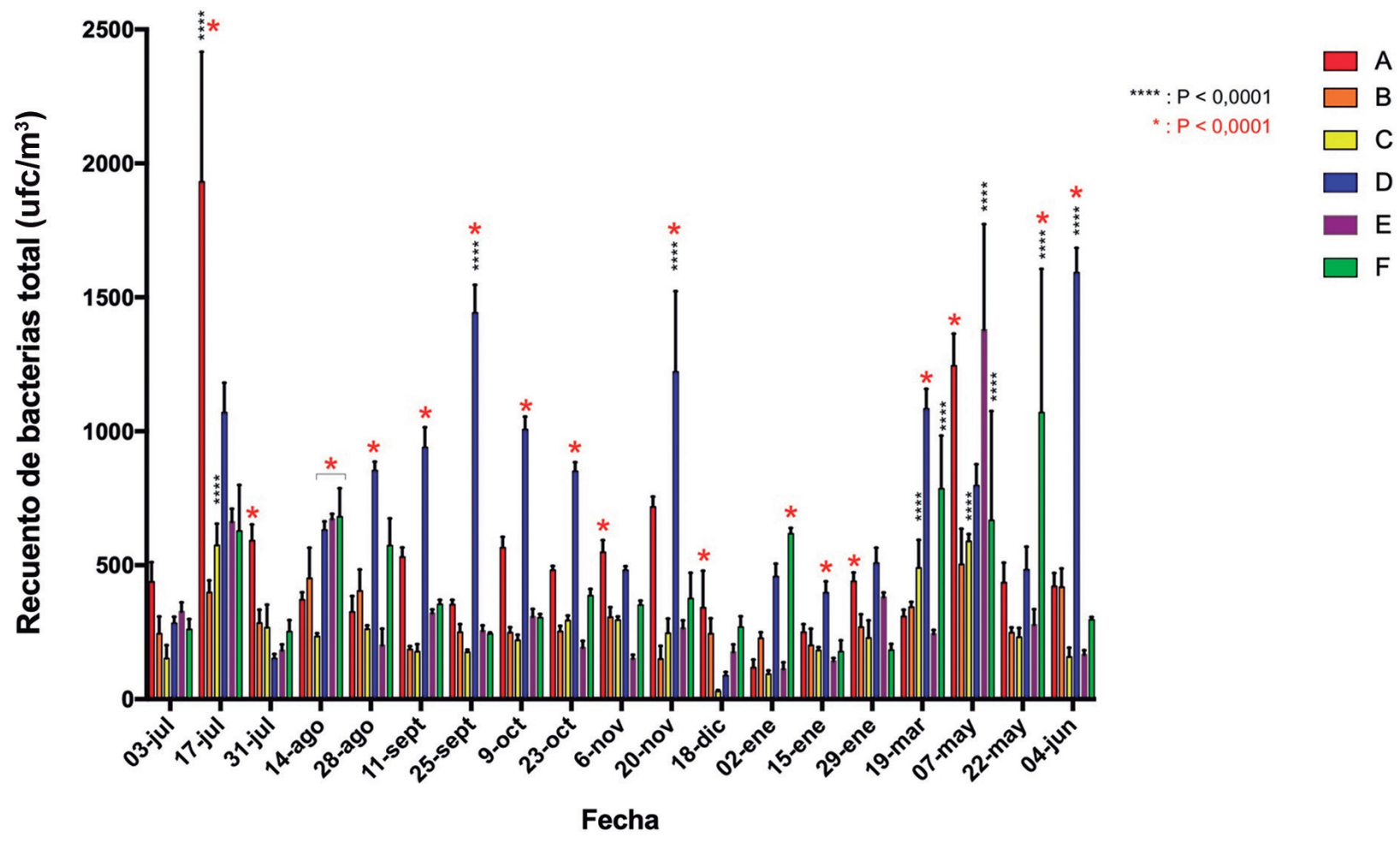

Figura 2. Recuentos bacterianos en muestras de aire en diferentes salas del CECOSF-Centinela de Talcahuano, entre los meses de julio de 2018 y junio de 2019 : A: sala de espera, B: sala de reuniones, C: sala de atención odontológica, D: sala de atención médica general, E: sala de atención de obstetricia/ginecología y F: sala de procedimientos generales. Las barras de error indican la desviación estándar producto de tres réplicas. Asteriscos negros: recuentos bacterianos significativamente mayores por sala. Asterisco rojo: Sala con recuento bacterianos significativamente mayor por fecha.

Tabla 1. Recuentos máximos y mínimos de bacterias/hongos $\left(u f c / \mathrm{m}^{3}\right)$ obtenidos en cada sala del CECOSF-Centinela de Talcahuano, entre los meses de julio de 2018 y junio de 2019

\begin{tabular}{ccccccc}
\hline Sala & A & B & C & D & E & F \\
Mínimo & $91 / 10$ & $96 / 10$ & $117 / 10$ & $104 / 10$ & $140 / 10$ & $204 / 10$ \\
Máximo & $2.376 / 153$ & $775 / 87$ & $846 / 61$ & $1.690 / 117$ & $1.658 / 74$ & $1.537 / 113$ \\
\hline
\end{tabular}

(A): sala de espera, (B): sala de reuniones, (C): sala de atención odontológica, (D): sala de atención médica general, (E): sala de atención de obstetricia/ginecología y (F): sala de procedimientos generales.

caso de las cepas bacterianas, se detectaron 11 géneros, donde mayoritariamente, correspondieron a bacterias grampositivas (96\%), destacando diversas especies de los géneros Bacillus (39\%) y Staphylococcus (33\%). La gran mayoría de las especies identificadas corresponde a bacterias sin importancia patogénica para el hombre. Sin embargo, es importante destacar que, aunque con muy baja frecuencia, se detectaron bacterias grampositivas patógenas como Staphylococcus aureus (2\%) y Kocuria rhizophila (5\%). En el caso de las bacterias gramnegativas, éstas fueron detectadas a una frecuencia muy baja (4\%), y correspondieron a las especies Pseudomonas oryzihabitans (3\%) y Acinetobacter lwoffii (1\%). Por otra parte, para los hongos se identificó un número menor de especies, perteneciendo $71 \%$ al género Aspergillus, con la siguiente distribución por especie: Aspergillus fumigatus (37\%), Aspergillus niger (23\%) y Aspergillus flavus (11\%), todos ellos patógenos para el hombre. 
Tabla 2. Valor máximo/mínimo de temperatura, humedad relativa y número de personas de cada sala del CECOSF-Centinela de Talcahuano, entre los meses de julio de 2018 y junio de 2019

\begin{tabular}{|c|c|c|c|c|c|c|}
\hline & A & B & C & D & $E$ & $F$ \\
\hline $\mathrm{T}\left({ }^{\circ} \mathrm{C}\right)$ & $26,4 / 16$ & $29,3 / 18,2$ & $24,1 / 19,3$ & $23,7 / 18,2$ & $24,4 / 18,5$ & $22,4 / 17,2$ \\
\hline HR (\%) & $70,1 / 32,8$ & $73,6 / 30,5$ & $74,6 / 50,3$ & $81,8 / 43,7$ & $75,2 / 46,3$ & $75,5 / 43,6$ \\
\hline $\mathrm{N}$ & $24 / 9$ & $8 / 2$ & $7 / 1$ & $7 / 1$ & $6 / 1$ & $8 / 1$ \\
\hline
\end{tabular}

(A): sala de espera, (B): sala de reuniones, (C): sala de atención odontológica, (D): sala de atención médica general, (E): sala de atención de obstetricia/ginecología y (F): sala de procedimientos generales, $(\mathrm{T})$ : temperatura, $(\mathrm{HR})$ : humedad relativa y $(\mathrm{N})$ : número de personas.

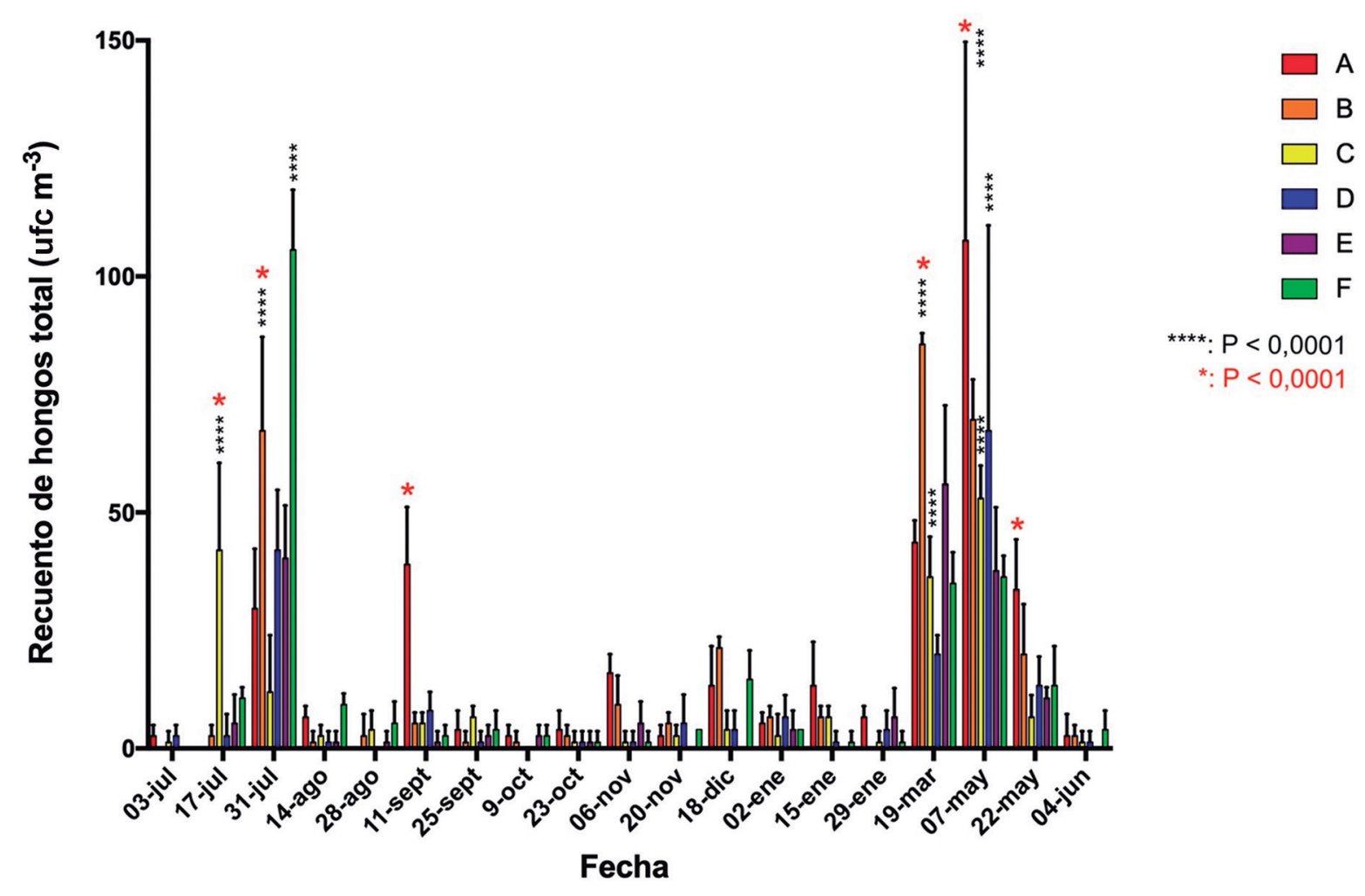

Figura 3. Recuentos de hongos obtenidos de muestras de aire en diferentes salas del CECOSF-Centinela de Talcahuano, entre los meses de julio de 2018 y junio de 2019 : A: sala de espera, B: sala de reuniones, C: sala de atención odontológica, D: sala de atención médica general, E: sala de atención de obstetricia/ginecología y F: sala de procedimientos generales. Las barras de error indican la desviación estándar producto de tres réplicas. Asteriscos negros: recuentos bacterianos significativamente mayores por sala. Asterisco rojo: Sala con recuentos bacterianos significativamente mayor por fecha.

\section{Discusión}

El ambiente interno de los centros de atención en salud constituye un hábitat natural para los microorganismos (bacteria, virus, hongos, parásitos), pudiendo ingresar éstos a través del aire que se introduce al abrir puertas y ventanas y así permanecer en las superficies y en el polvo ${ }^{12}$. Sin embargo, es reconocido, que la fuente más importante de microrganismos son las personas que ingresan a estos recintos, quienes los transportan, principalmente, en su piel y vías respiratorias ${ }^{13}$. Además, conociendo que el hombre inhala alrededor de 6-10 litros 
de aire por minuto, se hace necesario asegurar una buena calidad del aire, de tal forma de no afectar la salud de las personas que ingresan a esos recintos ${ }^{13}$. Así, la OMS ha publicado una serie de artículos enfocados en este tema, dando énfasis a que la calidad del aire, al interior de los recintos, es un aspecto cada vez más importante para la salud ocupacional y pública, puesto que afecta la salud de las personas, en especial aquellas más vulnerables, debido a su edad o estado de salud ${ }^{1,2}$.

Para cuantificar la carga microbiana en el aire, existen actualmente el método pasivo y el método activo; en ambos métodos se procede al conteo de las ufc, ya sea de bacterias y/o hongos ${ }^{6}$. En este estudio se empleó el método activo puesto que es el recomendado por el Instituto de Salud Pública de Chile ${ }^{8}$. Los recuentos máximos de bacterias y hongos, determinados en el aire al interior del CECOSF-Centinela en Talcahuano, Región del Biobío, variaron entre 2.376-775 ufc $/ \mathrm{m}^{3}$ para bacterias y 153-61 $\mathrm{ufc} / \mathrm{m}^{3}$, para los hongos. Al comparar estos resultados con otros, debe considerarse que la determinación de la carga microbiana es altamente dependiente del método utilizado para la toma de muestra, de la fecha de muestreo (variación estacional) y área geográfica. Todas estas variables hacen muy difícil establecer valores de referencia y sugieren que se debería realizar estudios en todos los ambientes en contacto con humanos, reforzando la importancia de este estudio ${ }^{14,15}$. Además, si los comparamos con algunos valores de referencia entregados en literatura científica, debemos tener en cuenta el sesgo de que mayoritariamente estos valores son para áreas hospitalarias limpias (pabellones quirúrgicos) $)^{3-6}$; evidentemente, el valor máximo de recuento bacteriano informado en este estudio está por sobre los valores informados en la literatura pertinente.

Por otra parte, se ha descrito que la presencia de bacterias y hongos en el aire, al interior de recintos cerrados, está influenciada por distintos factores ambientales entre los que se encuentran la T, la HR, la ventilación y aire acondicionado, los niveles de dióxido de carbono, concentración de oxígeno, polvos antropogénicos, tipo y materiales de construcción ${ }^{3,16}$. Aunque, sin duda, en recintos cerrados no industriales, la fuente más importante de microorganismos en el aire es la presencia humana ${ }^{13,15,16}$. En este estudio no se encontró correlación significativa entre los factores ambientales de T, HR y el recuento microbiológico $(\mathrm{P}>0,05)$. Estos resultados concuerdan con lo informado por Cho $\mathrm{y}$ cols., que relacionaron el recuento de microorganismos y variables ambientales, como T, HR y concentración de $\mathrm{CO}_{2}$, en recintos utilizados para población sensible o de alto riesgo como guarderías infantiles, hogares de ancianos y centros médicos en Corea del Sur ${ }^{3}$. Una de las razones que fundamentan los autores es la climatización de los ambientes; en nuestro estudio también las salas eran mantenidas con aire acondicionado a una tempe-
Tabla 3. Identificación, afinidad tintorial y frecuencia de bacterias aislados en CECOSFCentinela de Talcahuano

\begin{tabular}{lcc}
\hline Especie bacteriana & $\begin{array}{c}\text { Afinidad tintorial } \\
\text { (Gram }+/-)\end{array}$ & $\begin{array}{c}\text { \% de frecuencia de } \\
\text { la especie }\end{array}$ \\
Bacillus sp & + & 15 \\
Staphylococcus epidermidis & + & 12 \\
Staphylococcus saprophyticus & + & 10 \\
Bacillus licheniformis & + & 8 \\
Macrococcus epidermidis & + & 7 \\
Staphylococcus fleurettii & + & 6 \\
Kocuria rhizophyla & + & 5 \\
Bacillus simplex & + & 4 \\
Bacillus megaterium & + & 3 \\
Glutamicibacter protophormiae & + & 3 \\
Staphylococcus succinus & + & 3 \\
Bacillus cereus & + & 2 \\
Bacillus firmus & + & 2 \\
Bacillus mycoides & + & 2 \\
Exiguobacterium mexicanum & + & 2 \\
Kocuria rosea & + & 2 \\
Staphylococcus aureus & + & 100 \\
Auricoccus indicus & + & 1 \\
Bacillus asahii & + & 1 \\
Bacillus infantis & + & 1 \\
Bacillus safensis & + & 1 \\
Enterococcus faecium & + & 1 \\
Exiguobacterium sibiricum & + & 1 \\
Paenibacillus glucanolyticus & + & 1 \\
Planomicrobium glaciel & + & 1 \\
Pseudomonas oryzihabitans & + & 1 \\
Acinetobacter Iwoffii & + & 2 \\
Total & + & 1 \\
\hline
\end{tabular}

Tabla 4. Identificación, tipo y frecuencia de hongos aislados en CECOSF-Centinela de Talcahuano

\begin{tabular}{lcc}
\hline Hongo identificado & Tipo de hongo & $\begin{array}{c}\text { \% de frecuencia de } \\
\text { la especie }\end{array}$ \\
\hline Aspergillus fumigatus & Filamentoso & 37 \\
Aspergillus niger & Filamentoso & 23 \\
Aspergillus flavus & Filamentoso & 11 \\
\hline Neurospora sitophila & Filamentoso & 9 \\
\hline Rhodotorula mucilaginosa & Levadura & 9 \\
Meyerozyma guilliermondii & Levadura & 4 \\
Byssochlamys spectabilis & Filamentoso & 3 \\
\hline Phanerochaete chrysosporium & Filamentoso & 2 \\
\hline Talaromyces columbinus & Filamentoso & 2 \\
\hline Total & & 100
\end{tabular}


ratura de 21 a $22^{\circ} \mathrm{C}$, evitando que se generaran grandes cambios en los parámetros ambientales, en relación con las condiciones externas. Existen otros estudios que han informado una correlación significativamente positiva entre las variables ambientales de $\mathrm{T}$ y HR con el recuento de bacterias y hongos ${ }^{16}$. Esta similitud y divergencia entre nuestros resultados y los informados en la literatura científica podría deberse a que tanto la $\mathrm{T}$ como la HR que permiten el desarrollo los microorganismos varía entre las especies halladas en los distintos estudios ${ }^{17}$. Por ejemplo, las condiciones óptimas de HR para el crecimiento fúngico es $>75 \%{ }^{18}$, condición que en este estudio se presentó en un solo día, en las salas D, E y F (Tabla 3), lo que pudo haber contribuido a que la mayoría de los recuentos de hongos obtenidos fueron bajos. Más aún, se ha informado que, para materiales de construcción de edificios como el concreto, material de construcción del CECOSF- Centinela, el crecimiento de hongos se reduce a temperaturas entre $26-28^{\circ} \mathrm{C}$ y HR $<80 \%$ lo que coincide con las condiciones ambientales medidas en este estudio en la mayor parte del tiempo y casos (Tabla 3$)^{18,19}$. En este estudio no hubo correlación significativa, entre el recuento de bacterias y hongos y el número de personas en la mayoría de las salas, lo que puede ser explicado debido a que la cantidad de personas presentes fue relativamente constante $y$, en general, correspondía a personas citadas para su control médico o toma de exámenes.

Si bien el recuento de microorganismos es importante para determinar la calidad microbiológica del aire, más aún es la identificación de los microorganismos que se encuentran en él ${ }^{13-15}$. Se determinó que las bacterias grampositivas fueron predominantes en relación a las bacterias gramnegativas, siendo los géneros Bacillus y Staphylococcus los más abundantes. Bacillus, es un género de bacterias esporuladas donde se incluyen numerosas especies, mayoritariamente habitantes del ambiente y Staphylococcus, un género bacteriano que se encuentra en la piel y mucosas del hombre y otros animales, que suele ser transportada por las actividades comunes de éstos y por el polvo que se encuentra en suspensión o en el suelo ${ }^{13}$ Numerosos estudios coincidentes con estos resultados indican que las bacterias grampositivas corresponden al grupo más importante aisladas desde el aire ${ }^{5,13,19,20}$. Esto sería consecuencia de la gruesa pared celular presentada por las bacterias grampositivas que le permite ser más resistentes a las condiciones ambientales que las gramnegativas $^{21}$. En relación a las bacterias gramnegativas identificadas, éstas correspondieron a $P$. oryzihabitans y A. lwoffii, ambos bacilos gramnegativos no fermentadores, cuyo hábitat es principalmente ambientes húmedos ${ }^{22,23}$. En estos últimos años, $P$. oryzihabitans ha cobrado mayor importancia clínica siendo reconocida como patógeno oportunista en pacientes inmunodeprimidos, ocasionando diversos cuadros clínicos ${ }^{24-28}$ e incluso como agente causal de un brote ${ }^{29}$. Por lo tanto, a pesar de haber sido aislada en un número muy bajo, su presencia igual representa un riesgo para la salud de las personas que concurren a este centro de salud, considerando que pueden concurrir personas con factores predisponentes.

En el caso de los hongos, estos microorganismos son ubicuos en la naturaleza y dependiendo del género, se puede encontrar esporas, restos celulares, o células fúngicas en el aire ${ }^{13}$. En este estudio, el género Aspergillus, con las especies $A$. fumigatus, $A$. niger y $A$. flavus, fue el predominante, resultados que coinciden con literatura científica $^{6,13,20,30}$. Estos hongos corresponden a hongos filamentosos que producen abundantes y livianas esporas, lo que les permite mantenerse suspendidas y ser fácilmente trasportadas por el aire ${ }^{30}$. Las tres especies detectadas corresponden a hongos patógenos para el hombre, causantes de diferentes cuadros clínicos a nivel pulmonar. Sin embargo, se describe que A. fumigatus correspondería a la especie responsable de $90 \%$ de las infecciones en humanos, especialmente como agente causal de enfermedad fúngica invasora (EFI), entre la que destaca el cuadro clínico de aspergilosis invasora (AI) ${ }^{30-33}$. En Chile, un estudio realizado en 2017 estimó que la frecuencia de AI era de 1,7/100.000 individuos y, principalmente, en pacientes inmunocomprometidos ${ }^{32}$. Sin embargo, hay información que señala la posibilidad del desarrollo de estos cuadros clínicos en pacientes críticos, pero aparentemente inmunocompetentes ${ }^{31}$. Por otra parte, un estudio retrospectivo realizado en el período 20052015 informó un ligero incremento en la incidencia de EFI por hongos filamentosos, siendo Aspergillus el más frecuente $(53,6 \%)$, con una mortalidad a los 30 días de $39 \%$ y con una ubicación, principalmente, pulmonar ${ }^{33}$. Esto da cuenta de la importancia de la pesquisa de este microorganismo en el aire de estos centros de salud, considerando que es posible encontrar en estos lugares personas susceptibles a este tipo de infecciones, al inhalar esporas de estos hongos filamentosos. Considerando la importancia de la detección precoz de estos cuadros clínicos, en los últimos años se ha investigado en nuevos métodos de detección para diagnosticar precozmente esta enfermedad y así disminuir su letalidad ${ }^{34}$. Por otra parte, debe destacarse la importancia de $A$. flavus como segundo agente causante de infecciones sino-orbitales y oculares de países en desarrollo ${ }^{35}$.

\section{Conclusión}

El monitoreo del aire del CECOSF-Centinela de Talcahuano evidenció la presencia de bacterias y hongos con importancia en salud humana, en contraposición a lo recomendado por la OMS para el aire de recintos 
cerrados, en los que se incluyen estos centros de salud. Estos resultados deberían estimular la formulación de programas regulares de monitoreo de aire que permitan detectar la presencia de microorganismos potencialmente dañinos para la salud humana, minimizando el riesgo de exposición a estos microorganismos de las personas que concurren al centro de salud y el personal de salud que atiende en dichos establecimientos.

\section{Referencias bibliográficas}

1.- World Health Organization (WHO). WHO guidelines for indoor air quality - dampness and mould. Copenhagen, Denmark: WHO Regional Office for Europe; 2009, p 1-248. https://www.euro.who.int/_data/assets/pdf file/0017/43325/E92645.pdf?ua=1

2.- World Health Organization (WHO). WHO guidelines for indoor air quality: selected pollutants. 2010. http://www.euro.who.int/ data/assets/pdf_file/0009/128169/e94535.pdf.

3.- Cho E-M, Hyong J H, Park S H, Yoon D K, Goung S J N N, Lee M C. Distribution and influencing factors of airborne bacteria in public facilities used by pollution-sensitive population: a meta-analysis. Int J Environ Res Public Health 2019; 16(9): 1483. doi: 10.3390/ ijerph16091483.

4.- Stauning M T, Bediako-Bowan A, Andersen L P, Opintan J A, Labi A K, Kurtzhals J A L, et al. Traffic flow and microbial air contamination in operating rooms at a major teaching hospital in Ghana. J Hosp Infect 2018; 99: 263-70. doi.org/10.1016/j. jhin.2017.12.010.

5.- Cabo-Verde S, Almeida S M, Matos J, Guerreiro D, Meneses M, Faria T, et al. Microbiological assessment of indoor air quality at different hospital sites. Res Microbiol 2015; 166: 557-63. doi: 10.1016/j. resmic.2015.03.004.

6.- Veronesi L, Colucci M E, Napoli C, Castiglia P, Liguori G, Torre I, et al. Air microbial contamination in dental clinics: comparison between active and passive methods. Acta Biomed 2020; 91: 165-167. doi: 10.23750/abm. v91i3-S.9440.

7.- Atención Primaria. Servicio de Salud Metropolitano Norte. https://www.ssmn.cl/ atencion_primaria.ajax.php (Fecha de acceso a la información: 15 de marzo 2021).

8.- Chang C, Tseng L, Yang L. Microbial air contamination in an Intensive Care Unit. Int J Public Health Sci 2015; 4: 145-151. doi: 10.11591/. v4i3.4725.

9.- Valenzuela M T. Procedimiento muestreo microbiológico de aire. http://www.ispch.cl/sites/default/files/ documento tecnico/2011/01/Muestreo\%20 Microbiol\%C3\%B3gico\%20de\%20Aire.pdf (Fecha de acceso a la información: 20 de abril 2020).

10.- González-Rocha G, Muñoz-Cartes G,
Canales-Aguirre C B, Lima C A, Domínguez-Yévenes M, Bello-Toledo $\mathrm{H}$, et al. Diversity structure of culturable bacteria isolated from the Fildes Peninsula (King George Island, Antarctica): A phylogenetic analysis perspective. PLoS ONE 2017; 12(6): e0179390. doi: 10.1371/journal. pone. 0179390

11.- Martin K J, Rygiewicz P T. Fungal-specific PCR primers developed for analysis of the ITS region of environmental DNA extracts. BMC Microbiol 2005; 5: 28. doi: 10.1186/1471-21805-28.

12.- Altschul S F, Madden T L, Schäffer A A, Zhang J, Zhang Z, Miller W, et al. Gapped BLAST and PSI-BLAST: a new generation of protein database search programs. Nucleic Acids Res 1997; 25: 3389-402. doi: 10.1093/ nar/25.17.3389.

13.- Guo J, Xiong Y, Kang T, Xiang Z, Qin C. Bacterial community analysis of floor dust and HEPA filters in air purifiers used in office rooms in ILAS, Beijing. Sci Rep 2020; 10: 10401. doi: 10.1038/s41598-020-63543-1.

14.- González-Martin C. Airborne Infectious Microorganisms. Edited by Moselio Schaechter, Encyclopedia of Microbiology, 4th Ed., Academic Encyclopedia of Microbiology Press, 2019, pp. 52-60. doi.org/10.1016/B978-0-12809633-8.13002-X.

15.- Fujiyoshi S, Tanaka D, Maruyama F. Transmission of airborne bacteria across built environments and its measurement standards: a review. Front Microbiol 2017; 8: 2336. doi: 10.3389/fmicb.2017.02336.

16.- Božić J, Ilić P Ilić S. Indoor air quality in the hospital: the influence of heating, ventilating and conditioning systems. Braz Arch Biol Technol 2019; 62: e19180295. doi. org/10.1590/1678-4324-2019180295.

17.- Zavieh F, Mohammadi M, Vosoughi M, Abazari M, Raesee E, Fazlzadeh M, et al. Assessment of types of bacterial bio-aerosols and concentrations in the indoor air of gyms. Environ Geochem Health 2021. doi: 10.1007/ s10653-020-00774-1.

18.- Marques do Nascimento J, López, Andrade M, Anhezini L, Alves E. Airborne fungi in indoor hospital environments. Int J Curr Microbiol Appl Sci 2019; 8: 2749-72. doi: 10.20546/ ijcmas.2019.801.291.

19.- Wu H, Wong JWC. Current challenges for shaping the sustainable and mold-free hygienic indoor environment in humid regions.
Lett Appl Microbiol 2020; 70: 396-406. doi: 10.1111/lam.13291

20.- Zhang X, Liang J, Wang B, Lv Y, Xie J. Indoor air design parameters of air conditioners for mold-prevention and antibacterial in island residential buildings. Int J Environ Res Public Health. 2020; 17(19): 7316. doi: 10.3390/ ijerph17197316.

21.- Larrey E K, Laryea J N A, Kpordze S W, Saba C K S. Microbial load of indoor airborne bacteria and fungi in a teaching hospital in Ghana. African J Microbiol Res 2020; 14(3): 100-5. doi: 10.5897/AJMR2020.9297.

22.- Vollmer W, Didier Blanot D, de Pedro MA. Peptidoglycan structure and architecture. FEMS Microbiol Rev 2008; 32: 149- 67. doi:10.1111 j.1574-6976.2007.00094. x.

23.- Gutiérrez-Bustos D, Hernández-Marín A, Corrales-Ramírez L. Pseudomonas oryzihabitans: un microorganismo de creciente interés científico. NOVA 2009; 7(11): 104-11. https://doi.org/10.22490/24629448.1833.

24.- Rodríguez CH, Nastro M, Dabos L, Vay C, y Famiglietti A. Frecuencia de aislamiento y resistencia a los antimicrobianos de Acinetobacter spp. recuperadas de pacientes atendidos en un hospital universitario de la Ciudad Autónoma de Buenos Aires, Argentina. Rev Argent Microbiol 2014; 46(4): 320-4. doi: 10.1016/S0325-7541(14)70090-2.

25.- Keikha M, Soori T, Azadi D, Karami-Zarandi M, Daneshpazhooh M, Ali Rahdar H. The first report of Pseudomonas oryzihabitans in infection in a patient with hidradenitis suppurativa. Clin Case Rep 2019; 25: 1514-7. doi: $10.1002 / \mathrm{ccr} 3.2265$.

26.- Ochi F, Tauchi H, Nagai K, Moritani K, Ishii E. Pseudomonas oryzihabitans bacteremia in a child with peripheral T-cell lymphoma after allogeneic bone marrow transplantation. Pediatr Int 2018; 60(5): 486-8. doi: 10.1111/ped.13540.

27.- Owusu M, Owusu-Dabo E, Acheampong G, Osei I, Amuasi J, Sarpong N, et al. Pseudomonas oryzihabitans sepsis in a 1-year-old child with multiple skin rashes: a case report. J Med Case Rep. 2017; 11: 77. doi: 10.1186/s13256-017-1230-6.

28.- Hellou E, Artul S, Omari S, Taha M, Armaly Z, Nseir W. Non catheter-related bacteremia caused by Pseudomonas oryzihabitans in a patient undergoing hemodialysis. Hemodial Int 2014; 18: 711-3. doi: $10.1111 /$ hdi. 12150 . 
29.- Bhatawadekar S M. Community-acquired urinary tract infection by Pseudomonas oryzihabitans. J Global Infect Dis. 2013; 5: 824. doi: 10.4103/0974-777X.112274.

30.- Woo K S, Choi J L, Kim B R, Kim J E, Kim K H, Kim J M, et al. Outbreak of Pseudomonas oryzihabitans pseudobacteremia related to contaminated equipment in an emergency room of a tertiary hospital in Korea. Infect Chemother. 2014; 46: 42-4. doi: 10.3947/ ic.2014.46.1.42.

31.- Latgé J P, Chamilos G. Aspergillus fumigatus and aspergillosis in 2019. Clin Microbiol
Rev 2019; 33: e00140-18. doi.org/10.1128/ CMR.00140-18.

32.- Rabagliati R. Actualización en el diagnóstico y manejo de aspergilosis invasora en pacientes adultos. Rev Chilena Infectol. 2018; 35: 531-44. doi: 10.4067/s071610182018000500531.

33.- Álvarez Duarte E, Denning DW. Serious fungal infections in Chile. Eur J Clin Microbiol Infect Dis. 2017; 36: 983-6. doi: 10.1007/s10096-0172925-8.

34.- Valenzuela P, Rabagliati R, Legarraga P. Epidemiología de la enfermedad fúngica invasora por hongos filamentosos en el período 2005 a 2015, en un hospital universitario en Santiago, Chile. Rev Chilena Infectol. 2019; 36: 732-41. doi: 10.4067/S071610182019000600732 .

35.- Cruz R, Álvarez P, Provoste F, Ducasse K, Gonzalez M, Wilson G, et al. Enfermedad fúngica invasora (EFI) por hongos filamentosos en la Región de Valparaíso, Chile, desde la implementación del diagnóstico rápido de laboratorio. Rev Chilena Infectol. 2015; 32: 221-4. doi: 10.4067/S071610182015000300012 . 